\title{
A chemical-genetic strategy reveals distinct temporal requirements for SAD-I kinase in neuronal polarization and synapse formation Joanne SM Kim ${ }^{1,2}$, Brendan N Lilley33, Chao Zhang4, Kevan M Shokat ${ }^{4}$, Joshua R Sanes ${ }^{3}$ and Mei Zhen*1,2
}

\author{
Address: ${ }^{1}$ Department of Molecular Genetics, University of Toronto, Toronto, Ontario, M5S 1A8, Canada, ${ }^{2}$ Samuel Lunenfeld Research Institute, \\ Mount Sinai Hospital, Toronto, Ontario, M5G 1X5, Canada, ${ }^{3}$ Department of Molecular and Cellular Biology and Center for Brain Science, Harvard \\ University, Cambridge, MA 02138, USA and ${ }^{4}$ Howard Hughes Medical Institute and Department of Molecular and Cellular Pharmacology, \\ University of California, San Francisco, CA 94143, USA \\ Email: Joanne SM Kim - joanne.kim@utoronto.ca; Brendan N Lilley - lilley@fas.harvard.edu; Chao Zhang - zhang@cmp.ucsf.edu; \\ Kevan M Shokat - shokat@cmp.ucsf.edu; Joshua R Sanes - sanesj@mcb.harvard.edu; Mei Zhen* - zhen@mshri.on.ca \\ * Corresponding author
}

Published: 22 September 2008

Neural Development 2008, 3:23 doi:10.1186/1749-8/04-3-23
Received: 9 July 2008

Accepted: 22 September 2008

This article is available from: http://www.neuraldevelopment.com/content/3/I/23

(c) 2008 Kim et al; licensee BioMed Central Ltd.

This is an Open Access article distributed under the terms of the Creative Commons Attribution License (http://creativecommons.org/licenses/by/2.0), which permits unrestricted use, distribution, and reproduction in any medium, provided the original work is properly cited.

\begin{abstract}
Background: Neurons assemble into a functional network through a sequence of developmental processes including neuronal polarization and synapse formation. In Caenorhabditis elegans, the serine/threonine SAD-I kinase is essential for proper neuronal polarity and synaptic organization. To determine if SAD-I activity regulates the establishment or maintenance of these neuronal structures, we examined its temporal requirements using a chemical-genetic method that allows for selective and reversible inactivation of its kinase activity in vivo.
\end{abstract}

Results: We generated a PPI analog-sensitive variant of SAD-I. Through temporal inhibition of SAD-I kinase activity we show that its activity is required for the establishment of both neuronal polarity and synaptic organization. However, while SAD-I activity is needed strictly when neurons are polarizing, the temporal requirement for SAD-I is less stringent in synaptic organization, which can also be re-established during maintenance.

Conclusion: This study reports the first temporal analysis of a neural kinase activity using the chemical-genetic system. It reveals that neuronal polarity and synaptic organization have distinct temporal requirements for SAD-I.

\section{Background}

An emerging theme from recent studies of neural development is that many genes are employed repeatedly by different developmental processes. For example, morphogens such as WNTs are important not only for neural patterning but also for synaptogenesis $[1,2]$. Similarly, secreted factors such as UNC-6/netrin and sema- phorins are required for both axon guidance and synapse formation [3-7], and gamma-protocadherins promote both neuronal survival and synapse formation $[8,9]$. These molecules may serve multiple functions through a single or multiple genetic pathways during the different developmental processes. 
Distinguishing the multiple roles of a gene in neuronal development is often challenging because the developmental processes are highly interdependent: neurons that fail to polarize cannot form functional synapses, and conversely, failure in establishing synapses may lead to axon retraction and subsequent abnormalities in axon growth. Therefore, the involvement of a gene in later differentiation stages could be masked by an early-stage arrest. Addressing this issue with conventional genetic methods is not always possible. Gene knock-outs lead to an irreversible, often complete loss of gene function, and their mutant phenotypes are likely to reveal only the earliest roles during development. Temperature sensitive $(t s)$ or partial loss-of-function (LOF) genetic alleles are a commonly used alternative. However, ts alleles are available for only a very small number of genes, and partial LOF alleles may carry sufficient residual activity to obscure the functional identification. The conditional Cre-Lox recombination-induced gene knock-out system allows temporal and tissue-specific gene inactivation; but few Cre lines permit the tight temporal control required to analyze the neuronal differentiation events that transition from one stage to the next within a narrow window of time. Therefore, a complete but reversible inactivation strategy that allows for tight temporal control and tissue specificity would be an ideal approach to addressing this issue [10].

A chemical-genetic method that combines the specificity of genetics with the reversibility and temporal control of pharmacology has been developed for kinases. Initially identified as an inhibitor for the Src kinase [11], the adenine analog PP1 was modified to selectively bind genetically sensitized kinases [12]. In this method, the hydrophobic gate-keeper residue in the ATP-binding pocket of a kinase of interest is mutated to glycine or alanine (Figure 1A), creating extra space in the ATP-binding pocket. Exposing this mutant kinase to membranepermeable, non-hydrolyzable PP1 analogs containing a bulky moiety (for example, 1NA-PP1) allows for selective inactivation of the kinase of interest [13-19].

In this study, we adopted this chemical-genetic approach to study the temporal requirements for a serine/threonine kinase, SAD-1 (Synapses of amphids defective-1). Initially identified in Caenorhabditis elegans, SAD-1 is required for proper neuronal polarity and synaptic organization $[20,21]$. In sad-1 LOF mutants, synaptic vesicles and other presynaptic proteins are trafficked to both axons and dendrites, and vesicle clusters are abnormally diffuse at synapses. Its mammalian orthologs, SAD-A and SAD-B, also play multiple roles in neural development. Mouse SAD-A and SAD-B (also known as BRSK2 and BRSK1, respectively) function redundantly to regulate neuronal polarity in vivo $[22,23]$, and SAD-B regulates the release of synaptic neurotransmitters in cultured rat neurons [24].
The mechanism of SAD-1-mediated regulation of neuronal polarity and synaptic organization is not well-understood. Our recent study showed that SAD-1 physically interacts with a scaffolding protein, NAB-1 (Neurabin-1), and that this interaction is essential for regulating neuronal polarity but not synaptic organization [21]. These data suggest that SAD-1 regulates neuronal polarity through pathways that are distinct from those for synaptic organization. Whether SAD-1 is required for the establishment or maintenance of the two neuronal structures is unknown. To dissect the temporal requirements for SAD1 in neuronal polarity and synaptic organization, precise temporal inactivation of SAD-1 activity in vivo is needed. The kinase activity of SAD proteins is essential for their in vivo functions [20,23], making SAD-1 an ideal candidate for a chemical-genetic system that allows for inducible and reversible inactivation of kinase activity.

The chemical-genetic strategy has been used in yeast, Arabidopsis, Drosophila, mammalian cell lines, and mice [1319]. Here we report the first successful application of this method in C. elegans. Using this system, we discovered that the kinase activity of SAD-1 is required at developmental stages that coincide with the establishment of neuronal polarity and synaptic organization. However, while inactivating SAD-1 when neurons are establishing polarity and synapses led to irreversible defects in neuronal polarity, defects in synaptic organization were reversible and corrected by SAD-1 activity during maintenance. Therefore, these differentiation events have distinct temporal requirements for SAD-1 activity.

\section{Results \\ LI 23A SAD-I is functional and can be inactivated by a PPI analog in vitro}

To generate a SAD-1 variant that is sensitive to PP1 analogs, its gate-keeper residue, leucine 123, was mutated to an alanine (L123A; Figure 1A). The kinase activity of L123A SAD-1 was compared to that of the wild-type enzyme in an in vitro kinase assay using tau as a substrate [23] (Figure 1B). Upon activation by the LKB1 complex [25], both wild-type and L123A mutant enzymes were able to phosphorylate tau at S262. L123A SAD-1 was 30\% as active as the wild-type enzyme even at the highest ATP concentrations tested (Figure $1 \mathrm{~B}$ ). An alteration in activity of this magnitude is not uncommon for kinases with mutated gate-keeper residues, and in most cases the decrease in activity does not affect the ability of the kinase to properly function in vivo [13].

We next tested the sensitivity of wild-type and L123A SAD-1 to a PP1 analog, 1NA-PP1. The wild-type enzyme was unaffected by 1NA-PP1, but L123A SAD-1 was inhibited in a dose-dependent manner (Figure 1B). In sum- 

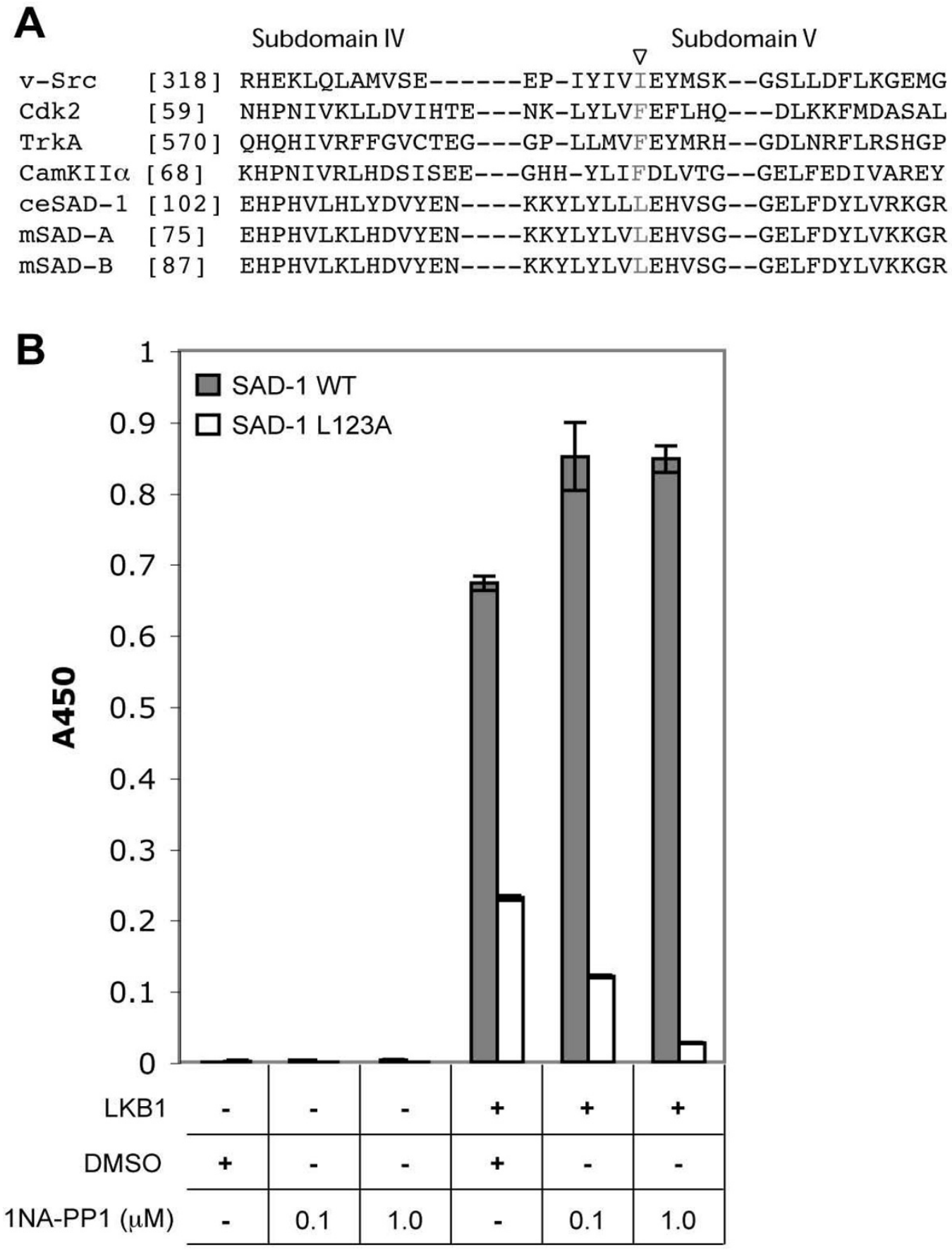

\section{Figure I}

LI 23A SAD-I is functional and can be inactivated with a PPI analog in vitro. (A) Alignment of $C$. elegans SAD-I and murine SAD kinases with other kinases successfully engineered using the chemical-genetic strategy. The gate-keeper residue is indicated in red. (B) In vitro kinase assay for SAD-I activity (described in Materials and methods). In the absence of LKBI, both wild-type (WT) and analog-sensitive (as) versions of SAD-I were inactive. Following treatment with LKBI, both versions became active towards tau [S262], but the kinase activity of the LI23A SAD-I was reduced 70\%. Inclusion of INA-PPI at the concentrations indicated caused significant inhibition of SAD- I as but not of wild-type SAD-I. $* * * p<0.00$ I, paired $t$-test, between DMSO- and INA-PPI-treated LI23A SAD-I samples. The $y$-axis indicates signal from ELISA assay for phospho-tau [S262]. Error bars indicate the standard error of the mean. 
mary, L123A SAD-1 retains kinase activity and is selectively sensitive to a PP1 analog.

\section{23A SAD-I is functional in vivo}

To determine if L123A SAD-1 can substitute for wild-type SAD-1 in vivo, we asked whether it can rescue neuronal polarity and synaptic organization phenotypes of sad-1 null mutants. The L123A mutation was introduced to a sad-1 construct that rescues defects in both neuronal phenotypes of sad-1 mutants $[20,21]$. This construct, $P_{\text {sad }-1^{-}}$ sad-1(L123A), was introduced into sad-1 (ky289) null mutants along with juIs1, a fluorescent marker of synaptic vesicles in GABAergic neurons $\left(P_{u n c-25-s n b-1:: g f p)}[26]\right.$. The juIs1 marker has been shown to be a reliable reporter for neuronal polarity [21] and synaptic organization $[20,21]$.

We first examined if L123A SAD-1 rescued the neuronal polarity defect in sad-1 mutants. In adult animals, the two classes of C. elegans GABAergic motoneurons, DDs and VDs, innervate dorsal and ventral body wall muscles, respectively. We observed vesicle trafficking in VD neurons by selectively eliminating the juIs1 expression in DD neurons (see Materials and methods). The polarity of VD neurons is reflected by the selective accumulation of the presynaptic juIs1 vesicle clusters, or puncta, in axonal processes along the ventral nerve cord and their exclusion from dendrites along the dorsal nerve cord in wild-type animals as previously described [21,27] (Figure 2Ai). In sad-1 mutants, on the other hand, vesicles are present in both ventral axons and dorsal dendrites (Figure 2Aii). This polarity defect was quantified by counting the number of ectopic, dorsal juIs1 puncta (Figure 2B, C). As previously reported [21], a 10-fold increase in ectopic juIs1 puncta was observed in sad-1 mutants relative to wild-type animals $\left(p=3.2 \times 10^{-6}\right)$. In the L123A SAD-1expressing sad-1 mutants, the number of ectopic juIs 1 puncta was decreased more than $50 \%\left(p=3.2 \times 10^{-6} \mathrm{com}\right.$ pared to sad-1 mutants; Figure 2B, C). This partial rescue may reflect the reduced kinase activity of L123A SAD-1 compared to the wild-type enzyme (Figure 1B). It is noteworthy, however, that wild-type SAD-1 also only partially rescued polarity defects in sad-1 null mutants $(p=3.2 \times$ $10^{-5}$ compared to sad-1 mutants; Figure 2C) [21], suggesting that the rescue of the sad-1 polarity phenotype is sensitive to the protein level typically over-expressed by transgenes.

We also asked whether L123A SAD-1 could rescue the synaptic organization defect in sad-1 mutants. In wild-type animals, juIs1 puncta in DD axons were round and discrete along the dorsal nerve cord, displaying the characteristic 'beads-on-a-string' morphology (Figure 2Di, Ei). In sad-1 mutants, juIs1 puncta appeared diffuse or smaller (Figure 2Dii, Eii). This defect in the juIs1 morphology correlates with a more diffuse distribution of synaptic vesicles in sad-1 mutants as shown by electron microscopy [20]. For a quantitative analysis of the juIs1 morphology, the width of each punctum was measured, and the population distribution of punctum widths was examined. As shown in Figure 2F, sad-1 mutants displayed a broader distribution of punctum widths, or 'lower kurtosis' of the distribution curve, than wild-type animals, consistent with the appearance of both diffuse and smaller puncta. In the L123A SAD-1-expressing sad-1 mutants, juIs1 puncta appeared more round and discrete (Figure 2E, F). Thus, L123A SAD-1 rescues synaptic organization as well as polarity defects in sad-1 mutants.

\section{LI23A SAD-I can be selectively inactivated by INA-PPI in vivo}

Next we asked whether L123A SAD-1 could be selectively inactivated by 1 NA-PP1 in vivo. We devised a liquid culture method using 96-well tissue culture dishes (Additional file 1), which allows for effective entry of 1NA-PP1 into animals through ingestion and also reduces the quantity of analog required. A dose-response curve was generated by exposing animals to different concentrations of 1NA-PP1 throughout their life and then assaying for polarity and synaptic organization phenotypes in adults (Figure 3 ). Whereas only partial inhibition was observed at lower concentrations, complete inhibition was observed at $33 \mu \mathrm{M}$ 1NA-PP1 ( $p=0.072$ compared to sad1 mutants; Figure 3A, C), and this concentration was used subsequently. This is comparable to, albeit slightly higher than, concentrations used for in vivo inactivation of PP1 analog-sensitive mammalian kinases $[14,16]$. Wild-type and L123A SAD-1-expressing sad-1 animals exposed to 33 $\mu \mathrm{M}$ 1NA-PP1 displayed slightly slower, but otherwise normal, growth. Exposing L123A SAD-1-expressing sad-1 animals to vehicle DMSO (Figure $3 \mathrm{~A}, \mathrm{Ci}$ ) or treating wildtype animals with 1NA-PP1 (Figure 3B) had no effect on neuronal polarity or synaptic organization.

These data show that L123A SAD-1 is both functional and selectively inactivated by 1NA-PP1 in vivo. We therefore hereafter refer to L123A SAD-1 as an analog-sensitive version of SAD-1 kinase (SAD-1as) and L123A SAD-1expressing sad-1 mutants as SAD-1as animals.

\section{SAD-I kinase activity is required for the establishment of neuronal polarity and synaptic organization}

Embryonically born DD neurons establish synapses with dorsal body wall muscles at the end of the first larval (L1) stage. VD neurons arise shortly thereafter and establish synapses with ventral body wall muscles [28] (Figure 4A). By the end of the second larval (L2) stage, polarization and synaptogenesis in these motoneurons are nearly complete. New synapses are added after the L2 stage, but they constitute less than $20 \%$ of the total number of synapses in adult animals [29]. We therefore defined the transition 
A

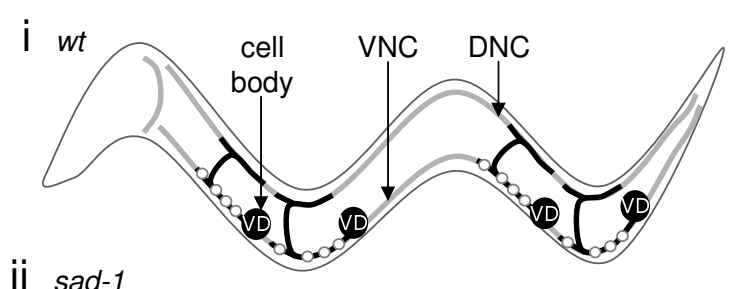

ii sad-1

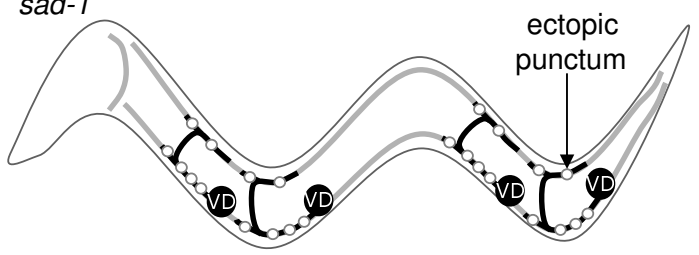

B

i

ii

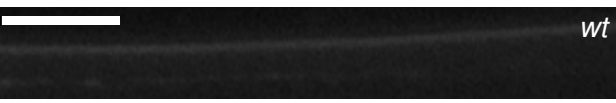

iii

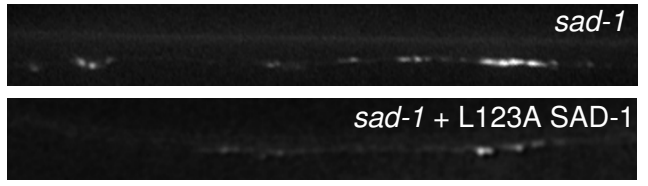

C

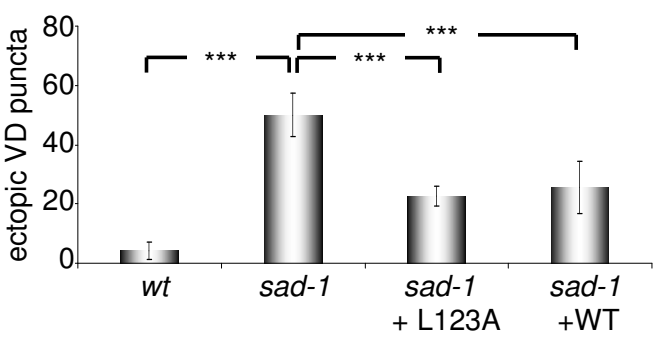

D

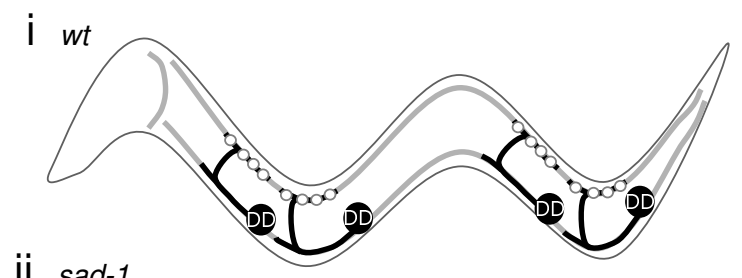

ii $s a d-1$

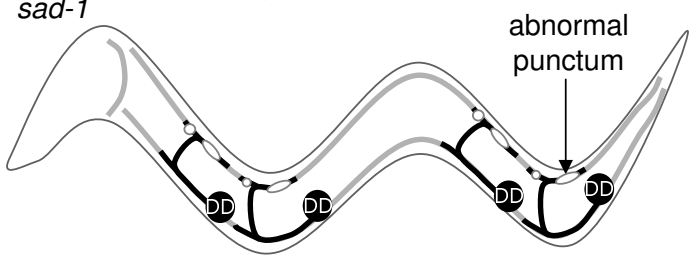

E

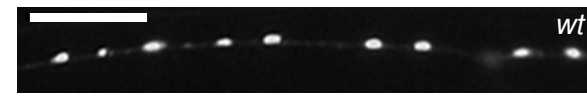

ii

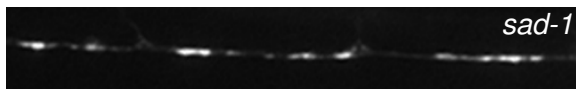

iii

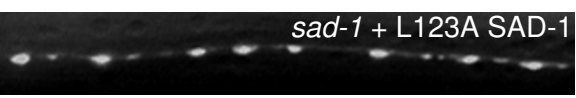

$\mathrm{F}$

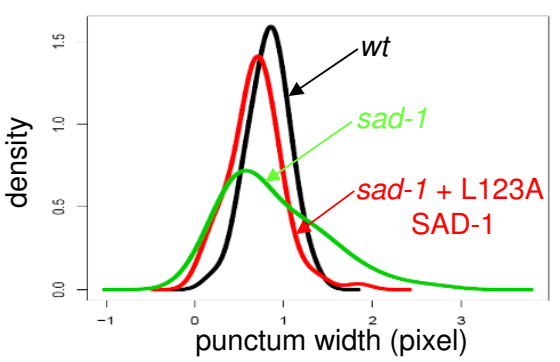

Figure 2

Figure 2

LI 23A SAD-I is functional in vivo. (A, B) Polarity phenotype. Axons of VD neurons innervate ventral body wall muscles, forming presynaptic terminals exclusively on the ventral side ( $\mathrm{Ai}$ ). In wild-type (wt) animals, only few, if any, ectopic juls I puncta are observed on the dorsal side (Bi). In sad-I LOF mutants, many ectopic juls I puncta are found (Aii, Bii). Expressing LI23A SAD-I partially rescued this phenotype, and fewer ectopic puncta were observed (Biii). DNC, dorsal nerve cord. VNC, ventral nerve cord. (C) Quantification of polarity phenotypes. Polarity defects were quantified by counting the number of ectopic juls I puncta per animal. Expressing LI23A SAD-I in sad-I mutants reduced their polarity defect more than 50\%, similar to expressing wild-type SAD-I. ***p $<0.00$ I, Wilcoxon rank-sum test. Error bars indicate standard deviations. (D, E) Synaptic organization phenotype. In wild-type animals, juls I puncta in DD neurons appear round and discrete along the dorsal nerve cord (Di, Ei). In sad-I mutants, diffuse or smaller puncta are observed (Dii, Eii). Expression of LI23A SAD-I rescued this phenotype and restored the juls / morphology (Eiii). (F) Quantification of synaptic organization phenotypes. Kernel density estimates of the punctum widths show a much wider distribution of punctum widths (that is, reduced kurtosis) in sad-I mutants compared to wild-type animals. LI23A SAD-I rescued this sad-I defect. Scale bar, $5 \mu \mathrm{m}$.

from the L2 stage to the L3 stage as the end of establishment and the beginning of maintenance for both polarity and synaptic organization in VD and DD neurons, respectively.

As noted above, life-long exposure of SAD-1as animals to 1NA-PP1 led to defects in neuronal polarity and synaptic organization (Figure 3). To ask whether the kinase activity of SAD-1 is required for the establishment or maintenance of neuronal polarity, we exposed SAD-1as animals to 1NA-PP1 during early larval stages or later (Figure 4B) and counted ectopic juIs1 puncta in adults (Figure 4C). Inhibiting SAD-1as throughout establishment and not maintenance was sufficient to abolish its rescuing effects on the polarity defect (Figure 4Bi, C). Moreover, inhibiting SAD1 as for as few as 30 hours during L1 and L2 was sufficient 
A

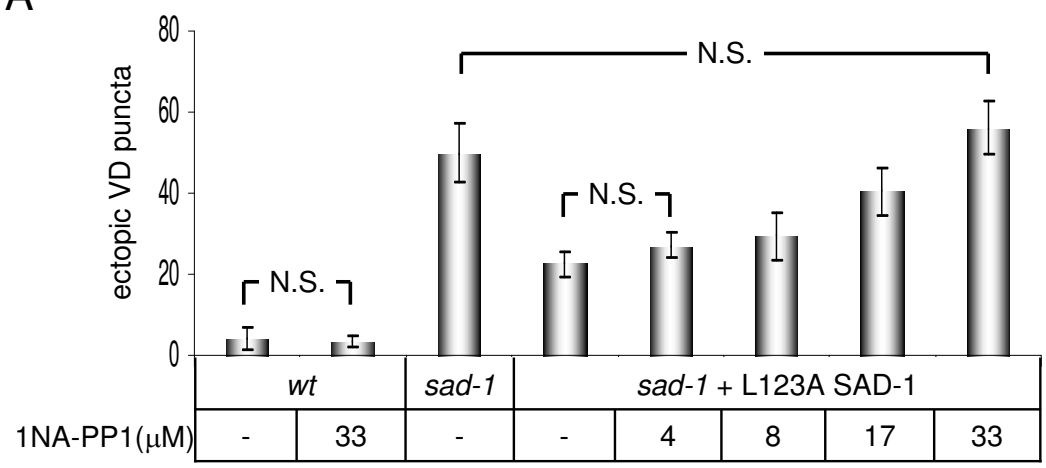

C

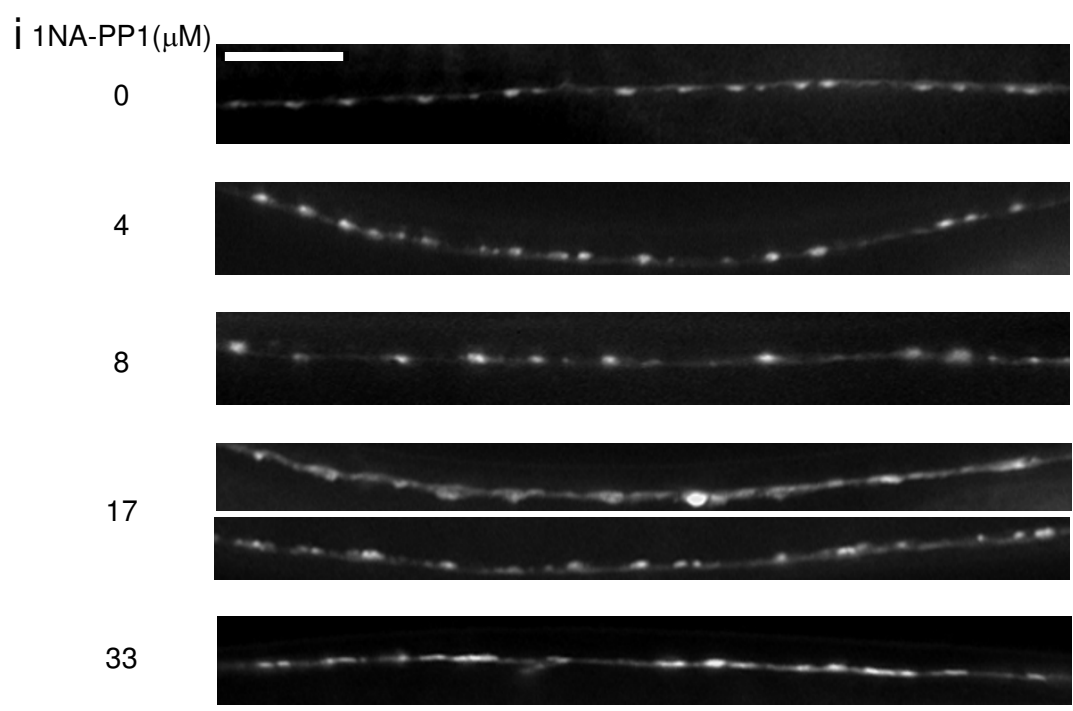

B

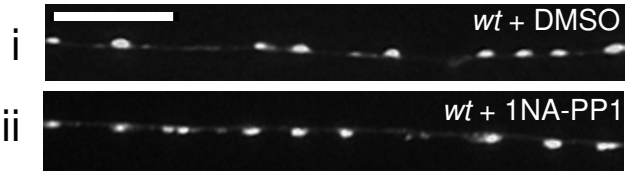

iii

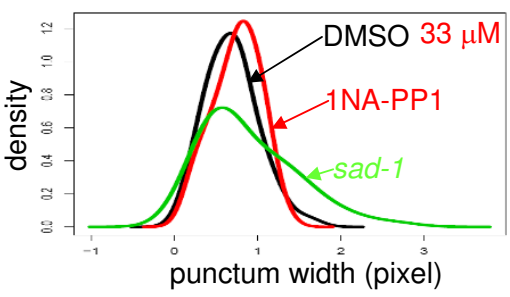

ii

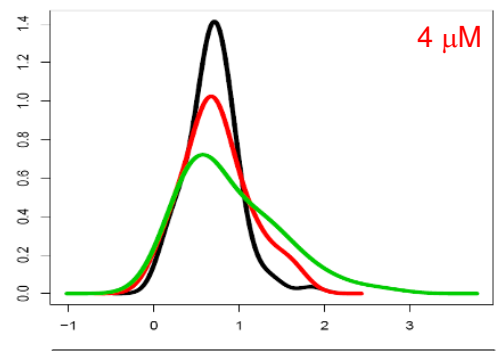

iii

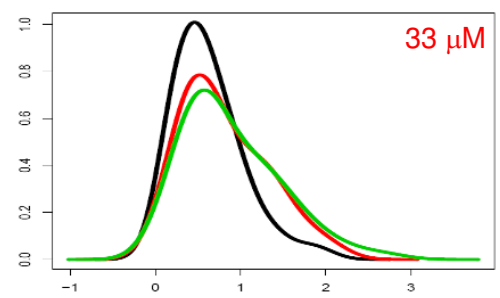

Figure 3

\section{Figure 3}

INA-PP I-induced SAD-I as inactivation is dose-dependent. (A) Dose-response in polarity phenotype. LI23A SAD-Iexpressing sad-I animals were exposed to various concentrations of INA-PPI, and the number of ectopic juls I puncta on the dorsal side of each animal was counted. At $4 \mu \mathrm{M}$, the level of polarity defect was similar to the animals exposed to DMSO. At $8 \mu \mathrm{M}$ and $17 \mu \mathrm{M}$, the inhibiting effects were stronger but incomplete. At $33 \mu \mathrm{M}$, complete inhibition of SAD-I as was observed. INA-PPI had no effect on the polarity phenotype of wild-type (wt) animals. N.S., not significant, Wilcoxon rank-sum test. Error bars indicate standard deviations. $(B, C)$ Dose-response in synaptic organization phenotype. At $33 \mu$, INA-PPI had no effect on the juls I morphology of wild-type animals (compare Bi and ii; quantified in Biii). LI23A SAD-I-expressing sad-I animals were exposed to various concentrations of INA-PPI, and the juls I morphology on the dorsal side of the animals was observed (Ci). At $4 \mu \mathrm{M}$ and $8 \mu \mathrm{M}$, most animals appeared normal. At I7 $\mu \mathrm{M}$, while some juls I puncta appeared defective (upper panel), others were normal (lower panel). At $33 \mu \mathrm{M}$, the rescuing effects of SAD-I as were inhibited in all animals. Quantification and density estimates of the punctum widths show that while $4 \mu$ I INA-PPI had little effect (Cii), $33 \mu M$ INA-PPI completely inhibited LI23A SAD-I (Ciii). All density graphs show punctum widths on the $x$-axis and density on the $y$-axis. Scale bar, $5 \mu$ m.

to elicit the same level of polarity defect (Figure 4Bii, C). Conversely, an exposure to 1NA-PP1 throughout maintenance had little effect on the polarity phenotype of SAD1as animals (Figure 4Biii, C). These results suggest that SAD-1 activity is required for the establishment, but not the maintenance, of neuronal polarity.
In contrast to the dramatic effects of early larval inhibition on adult neuronal polarity, inactivating SAD-1as for either three days or 30 hours in early larvae had little effect on the juIs1 morphology in adults (Figure 4B, D). Diffuse puncta were observed occasionally after three days of inactivation (Figure 4Di), but this subtle abnormality was not quantitatively significant (Figure 4Div). 
A

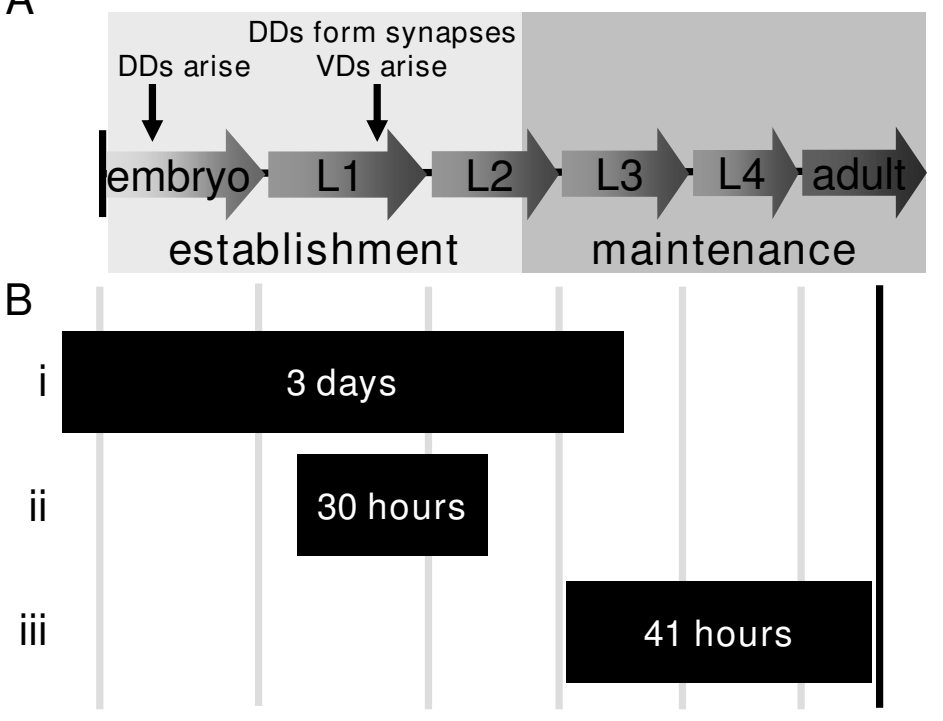

C

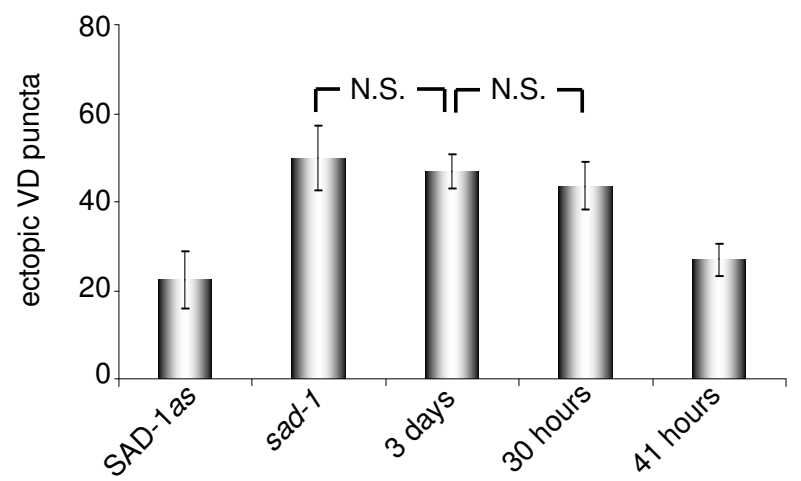

D

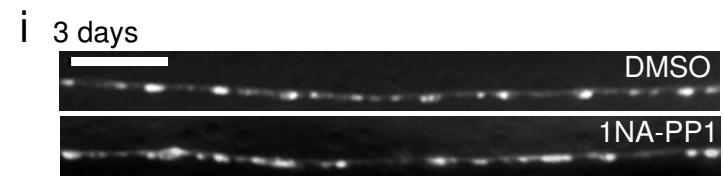

ii 30 hours

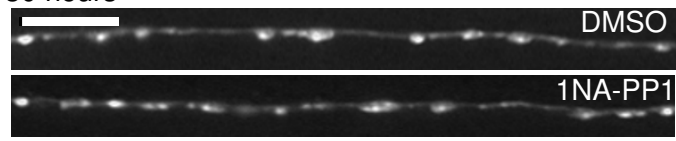

iii 41 hours

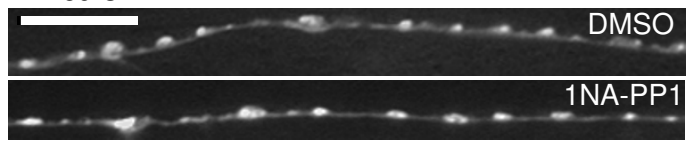

iv 3 days

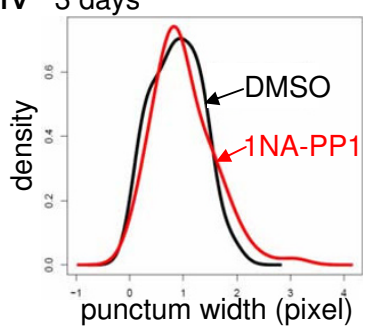

vi 41 hours

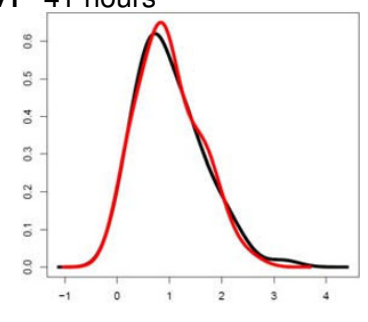

V 30 hours

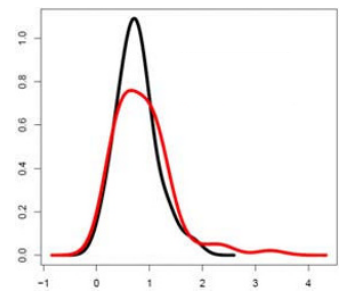

Figure 4

\section{Figure 4}

SAD-I kinase is required during the establishment of neuronal polarity but largely dispensable for the maintenance of neuronal polarity and synaptic organization. (A) Development of the GABAergic motoneurons. DD neurons arise embryonically and form synapses in late LI. VD neurons arise concomitantly. Whereas the establishment of DD and VD neurons is mostly complete by the end of L2, they are maintained for the C. elegans lifetime. (B) Two early larval and one late exposure. SAD-I as animals were exposed to INA-PPI throughout establishment (Bi), partially during establishment (Bii), or during maintenance (Biii) (filled bars) and observed at an adult stage (solid line). (C) Polarity phenotypes following the exposures. The three-day establishment exposure abolished the rescuing effects of SAD-I as, and the 30-hour duration was sufficient to replicate this full establishment exposure. The $4 \mathrm{I}$-hour exposure had no effect. N.S., not significant, Wilcoxon ranksum test. Error bars indicate standard deviations. (D) Synaptic organization phenotypes following the exposures. Animals treated with INA-PPI throughout establishment for three days occasionally displayed diffuse julsI puncta (bottom panel) compared to DMSO-treated animals (upper panel) (Di). The 30-hour and 4I-hour durations had no effect on the SAD-Ias-induced rescue (Dii-iii). Quantification and density estimates of the punctum widths show that there is no obvious difference between DMSO (black) and INA-PPI (red) treatments for any of the three exposures (Div-vi). All density graphs show punctum widths on the $\mathrm{x}$-axis and density on the $\mathrm{y}$-axis. Scale bar, $5 \mu \mathrm{m}$.

These results suggest at least two possibilities. SAD-1 may be required solely for the maintenance of already-established synaptic organization and not for synapse formation. However, this is unlikely as exposing animals selectively during maintenance did not affect the juIs1 morphology (Figure 4Biii, Diii, vi). Alternatively, SAD-1 kinase activity during the maintenance stage may be sufficient to re-establish synaptic organization. To test this possibility, we treated SAD-1as animals with 1NA-PP1 either throughout establishment until the end of the L2 stage or during the short 30-hour duration of DD synapse formation and observed their juIs1 morphology immedi- 
ately after treatment (Figure 5Aii, iii). Indeed, juIs1 puncta appeared diffuse immediately after treatment, suggesting that inhibiting SAD-1as during synaptogenesis abolished its rescuing effects on vesicle clustering (Figure 5B). Therefore, the re-activation of SAD-1as during maintenance following the early exposures to 1NA-PP1 led to restoration of the juIs1 morphology in adults (Figures 4Dii-iii, 5Bi). This phenomenon was specific to synaptic organization and not neuronal polarity, as early larval exposures to 1NA-PP1 followed by reactivation of SAD-1as did not correct the vesicle trafficking defect (Figure 4C). Thus, while
SAD-1 activity is required strictly when neurons are polarizing, it can promote synaptic organization during both establishment and maintenance.

\section{Distinct temporal requirements exist for SAD-I kinase activity during neuronal development}

Our study demonstrates the first application of the chemical-genetic system in C. elegans. To further test our findings from using this new methodology, we employed an independent method. Instead of inactivating SAD-1, we utilized a heat-shock (HS) inducible system to express
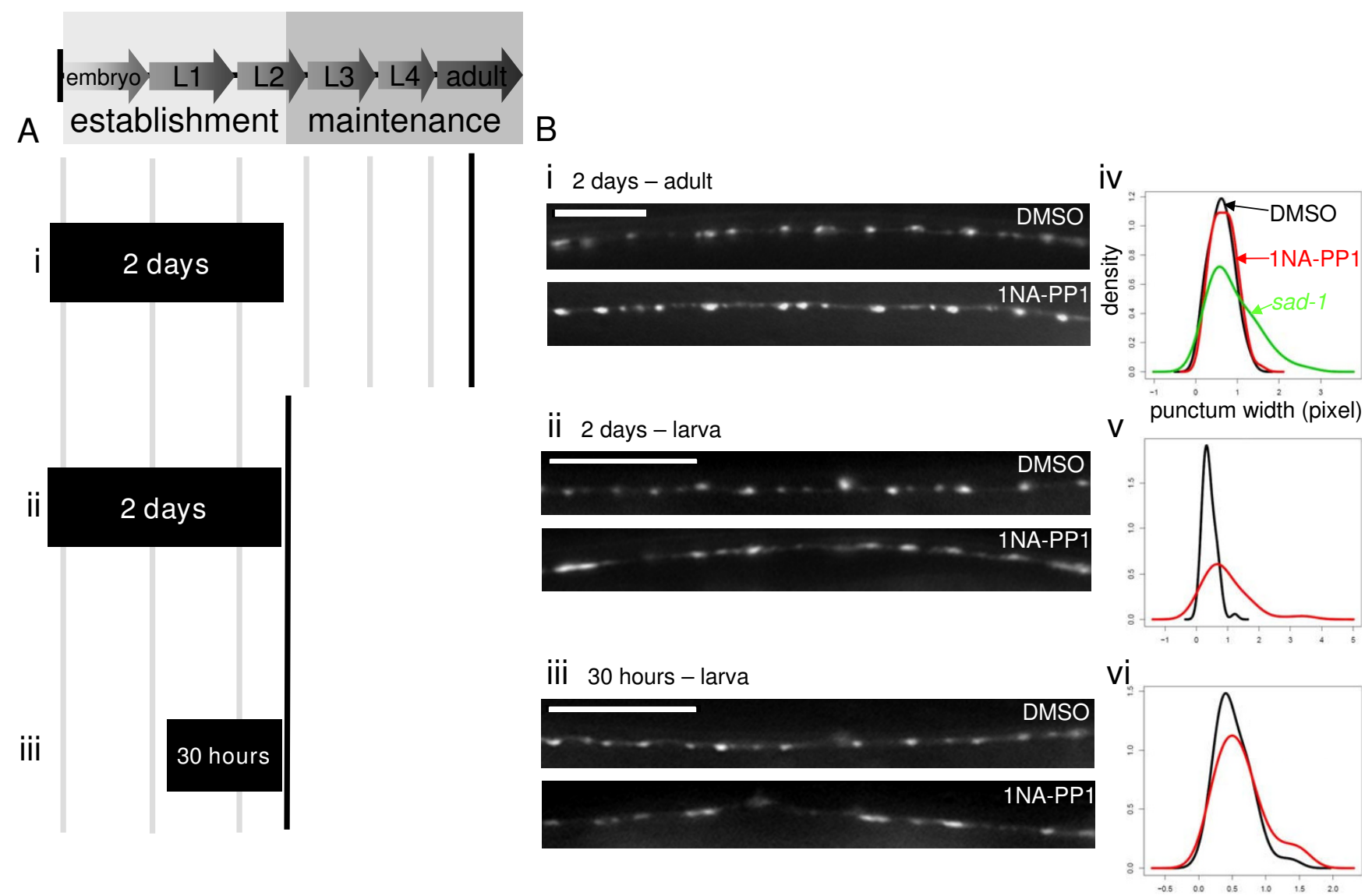

\section{Figure 5}

SAD-I kinase establishes synaptic organization. (A) Developmental exposures. SAD-I as animals were exposed to INAPPI throughout establishment (Ai-ii) or partially during establishment (Aiii) (filled bars). Observations were made at an adult stage $(\mathrm{Ai})$ or immediately following the exposures (Aii-iii) (solid lines). (B) Synaptic organization phenotypes following the exposures. The two-day establishment exposure to INA-PPI had no effect on the SAD-Ias-induced rescue observed at the adult stage $(\mathrm{Bi})$. However, when SAD-I as animals were observed immediately after treatment, the rescuing effects of SAD-Ias were abolished (Bii-iii). Quantification and density estimates of the punctum widths show that in the adult stage, DMSO (black) and INA-PPI (red) treatments appeared identical and rescued compared to sad-I mutants (Biv). In contrast, immediately after INA-PPI treatment, obvious changes in the distribution of the punctum widths were observed (Bv-vi). All density graphs show widths on the $x$-axis and density on the $y$-axis. Scale bar, $5 \mu \mathrm{m}$. 
wild-type SAD-1 in sad-1 null mutants. If SAD-1 determines neuronal polarity solely in developing neurons, expression of SAD-1 after the L2 stage would not rescue the neuronal polarity defect in adults. In contrast, if SAD1 could re-establish synaptic organization in the maintenance stage, expression of SAD-1 after the L2 stage should rescue the synaptic morphology defect in adults.

We used a promoter that drives ubiquitous expression in C. elegans upon HS (pPD118.26) [30]. To confirm its expression in the nervous system and examine the stability of SAD-1, an amino-terminal green fluorescent protein (GFP) fusion of SAD-1 under the HS protein promoter $\left(P_{H S^{-}}\right.$gfp::sad-1) was generated. Only after HS was strong GFP expression observed, confirming that GFP::SAD-1 expression was tightly regulated by temperature in our experimental system (described in Materials and methods). In the nervous system, GFP::SAD-1 expression was observed along the processes and in the cell bodies of body neurons (Figure 6A).

We generated a HS-inducible SAD-1 construct $\left(P_{H S}-\right.$ sad-1) and transformed it into sad-1 (ky289) null mutants. SAD1 was expressed by HS at the end of the L2 stage, and the polarity and synaptic organization phenotypes were observed in adults (Figure 6B, C). No rescue in the polarity defect was observed ( $p=0.51$ between with and without HS; Figure 6B). In contrast, following HS, the juIs1 morphology was rescued, appearing more round and discrete (Figure 6Cii, 6iv). This was not a result of HS treatment itself as the juIs1 morphology in sad-1 mutants was unaltered by HS (Figure 6Ci, iii). Therefore, consistent with our findings from the chemical-genetic system, the expression of SAD-1 in the maintenance stage rescued synaptic organization but not neuronal polarity defects (Figure 7).

\section{Discussion}

We report here the first successful application of the chemical-genetic system to analyzing the temporal requirements of a kinase in C. elegans. A PP1 analog-sensitive version of SAD-1 kinase, SAD-1as, was functional and sensitive to 1NA-PP1-mediated inhibition, both in vitro and in vivo. Using this system, we temporally inactivated SAD-1 kinase during different stages of the C. elegans life-cycle and found that SAD-1 activity is necessary in establishing both neuronal polarity and synaptic organization but dispensable for their maintenance (Figure 7). However, while SAD-1 activity is required strictly during a narrow window of time to establish neuronal polarity, its activity is not temporally restricted to the establishment stage and sufficient to re-establish synaptic organization in later stages. Our study thus reveals temporally distinct requirements for SAD-1 activity during neuronal differentiation.

\section{A chemical-genetic approach in analyzing kinase functions in C. elegans}

We showed that SAD-1as can be generated by mutating the gate-keeper residue, allowing inducible, fast, and reversible inhibition of its kinase activity with a specific small-molecule inhibitor. In this study, a small-volume liquid culture system was devised that offered several advantages over conventional plate cultures (Additional files 1 and 2; described in Materials and methods). The small volumes of the liquid culture required only microgram quantities of 1NA-PP1, reducing the quantity of inhibitor used 30-fold. The liquid culture also facilitated efficient administration of the membrane-permeable inhibitor through feeding.

PP1 analogs effectively blocked the activity of as-kinases at nanomolar concentrations in yeast and mammalian cell cultures $[13,16]$. In our study, micromolar-range 1NAPP1 was required to achieve complete inhibition of SAD1 as (Figure 3). This is likely caused by the poor permeability of C. elegans cuticles, only allowing entry through feeding. Micromolar concentrations of PP1 analogs were also required to inhibit mammalian as kinase activities in intact animals through oral administration $[14,16]$. Therefore, the efficacy of 1NA-PP1 in C. elegans is comparable to that in mice in vivo.

The kinetics of 1NA-PP1 entry in C. elegans is unknown. However, we do not think slow kinetics of entry was a confounding factor in our analyses because the shortest exposure time of 30 hours was sufficient for 1NA-PP1 entry and SAD-1as inactivation (Figure 7Av, vii). Indeed, a similar PP1 analog, 1NM-PP1, has been shown to cross the murine blood-brain barrier within minutes [14]. We also do not believe differential susceptibilities to 1NA-PP1 entry existed during different larval stages of the C. elegans life-cycle because the phenotypic difference in synaptic organization between the lifetime exposure (Figure 7Aii) and early larval exposures (Figure 7Avi, vii) suggests that there was sufficient 1NA-PP1 entry and inhibition of SAD1as across the larval stages.

Although the chemical-genetic system has proven to be quite a versatile and effective tool for regulating the activity of different kinases in yeast and cell culture studies, its application to intact animal models has been limited. Many kinase families display strong functional conservation across different animal species. The ease of genetic manipulation, simple physiology, and short developmental cycle make $C$. elegans an attractive target for using chemical genetics to temporally dissect the functional requirements of kinases. Our protocol may be applicable to other kinases or even other proteins containing ATPbinding domains [31] in C. elegans. 
A

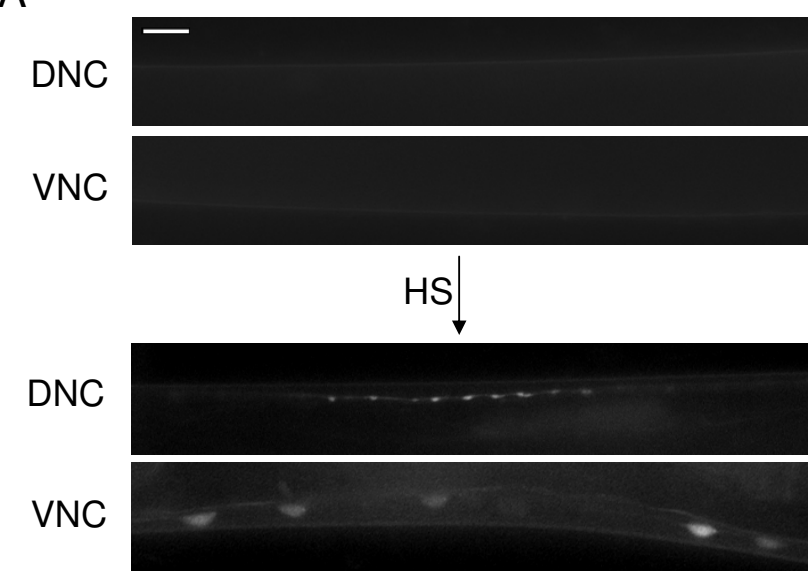

B

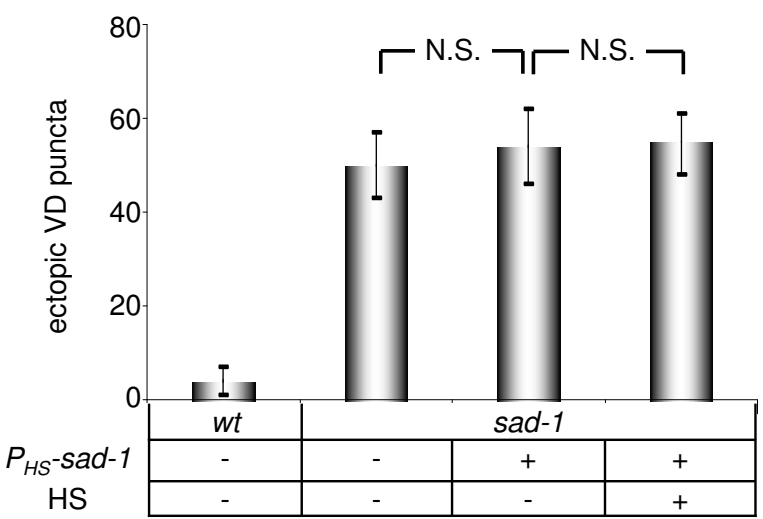

C
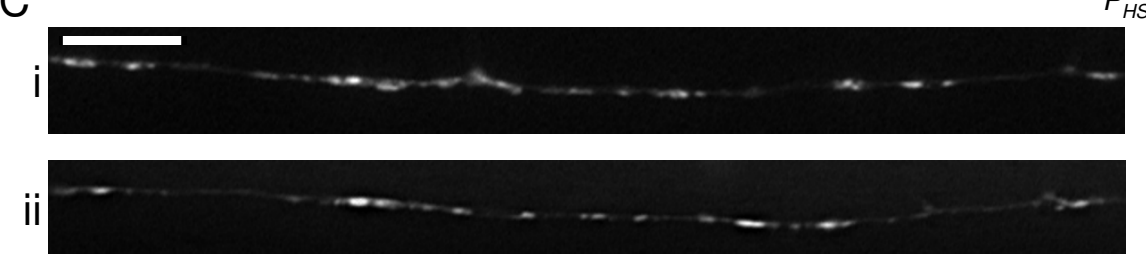

iii
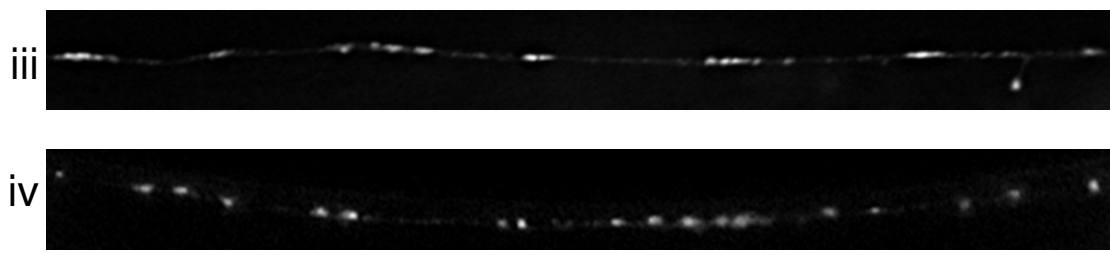

$P_{H S}$ Sad-1 HS

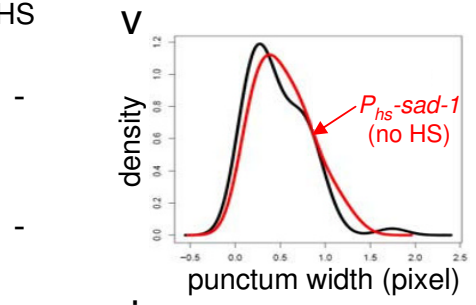

vi

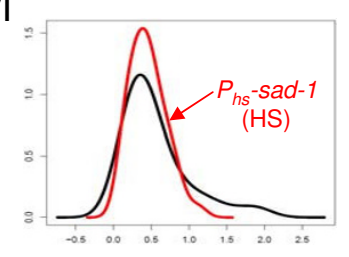

\section{Figure 6}

Expression of SAD- I in sad- I mutants during maintenance rescues defects in synaptic organization but not in neuronal polarity. (A) GFP::SAD-I expression in C. elegans nervous system following heat shock (HS). No GFP was detected in animals reared at $22^{\circ} \mathrm{C}$ (top panels). However, upon HS, strong GFP fluorescence was detected in various neurons, including those along the body (bottom panels). GFP fluorescence was seen along processes on the dorsal nerve cord (DNC) as well as in cell bodies on the ventral nerve cord (VNC). (B) Polarity phenotype following post-L2 expression of SAD-I. Expression of SAD-I during maintenance had no rescuing effects on the VD polarity phenotype. N.S., not significant, Wilcoxon rank-sum test. Error bars indicate standard deviations. (C) Synaptic organization phenotype following post-L2 expression of SAD- I. Diffuse juls puncta were observed in sad-I mutants before ( $\mathrm{Ci}$ ) and after (Ciii) HS. Expression of SAD-I during maintenance rescued the juls I morphology phenotype (compare Cii and Civ). Quantification and density estimates of the punctum widths show no differences between sad-I mutants (black) and HS construct-containing mutants (red) (Cv). However, upon HS, changes in the distribution of punctum widths can be observed (Cvi). Both density graphs show widths on the $x$-axis and density on the $y$ axis. Scale bar, $5 \mu \mathrm{m}$.

\section{Distinct temporal requirements for SAD-I activity in neuronal polarization and synapse formation}

We previously demonstrated that SAD-1 regulates neuronal polarity and synaptic organization through different genetic pathways [21]. Using inducible modulation of protein activity (as) and of mRNA expression (HS), we have now demonstrated that the neuronal structures also display different temporal requirements for SAD-1 activity.

The molecular basis for the temporal distinction between neuronal polarity and synaptic organization is unknown. The simplest explanation is that SAD-1 targets different substrates during polarization and synapse formation. 


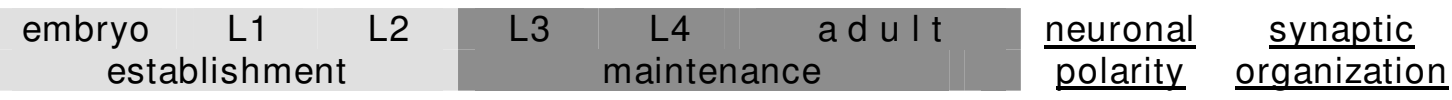

A

(as)

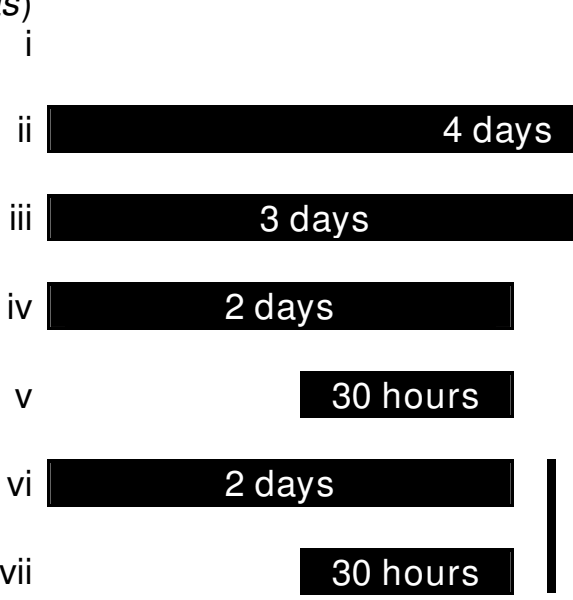

viii

41 hours

B (HS)

29 hours

Figure 7

Summary of neuronal phenotypes following different temporal exposures to INA-PPI or heat shock. (A) INAPPI exposures of SAD-I as animals (italics). (B) Heat shock expression of wild-type SAD-I in sad-I mutants (bold). Filled bars indicate no SAD-I. Solid lines represent points of observation. +, normal; -, defective; N.D., no data. Slower growth was observed in liquid culture than on agar (compare Aviii and B), likely caused by lower levels of oxygen in liquid.

While the substrates involved in neuronal polarization may only be present or active during the establishment stage, those involved in synapse formation may be present throughout establishment and maintenance. The mammalian orthologs of SAD-1, SAD-A and SAD-B, have been shown to regulate neuronal polarity through phosphorylation of tau, a microtubule associated protein [22,23], and subsequent promotion of microtubule dynamics during axon extension [32]. As an activator of microtubule dynamics, SAD-1 would define neuronal polarity only during the establishment stage. At synapses, the diffuse vesicle clustering in sad-1 mutants may reflect a failure in regulating vesicle release at the active zone or vesicle retrieval at the periactive zone. SAD-1 might phosphorylate distinct substrates that are required for organizing the endo- and exo-cytosis apparatus or for facilitating the interactions between vesicles and active zone/periactive zone regions.
To further our understanding of SAD-1, it is critical to identify all of its physiological substrates. The chemicalgenetic system has been used to identify substrates of kinases in in vitro assays $[33,34]$. Exploiting our SAD-1as system with substrate-labelling analogs may facilitate the identification of substrates and provide insights to the mechanism of SAD-1 in the future.

\section{Materials and methods Strains}

All C. elegans strains were cultured at $22^{\circ} \mathrm{C}$ using standard procedures on Nematode Growth Medium [35] with OP50 Escherichia coli as the food source.

juIs1 ( $\left.P_{\text {unc-25 }}-s n b-1:: g f p\right)$ has been described previously

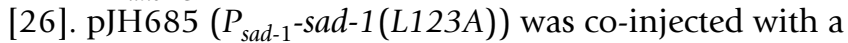
$P_{\text {odr-1 }}$-gfp marker into sad-1 (ky289); juIs1 animals and integrated into the C. elegans genome by UV irradiation. 
The integrants were out-crossed four times against wildtype N2 animals to generate hpIs89. From this, hpIs89; sad1 (ky289); juIs1 was generated and termed 'SAD-1as animals'. pJH73 $\left(P_{\text {unc-115-sad-1 }}\right)$ was co-injected with a $P_{o d r-1}{ }^{-}$ gfp marker into sad-1 (ky289); juIs1 animals to generate sad-1 (ky289); juIs1; hpEx1043 animals. pJH1360 ( $P_{H S}{ }^{-s a d-}$ 1) was co-injected with a $P_{o d r-1}$ gfp marker into sad-1 (ky289); juIs1 animals to generate sad-1 (ky289); juIs1; $h p$ Ex1431 animals. pJH1368 ( $\left.P_{H S^{-}} g f p:: s a d-1\right)$ was coinjected with a $P_{\text {lin-15 }}$-lin-15 into lin-15 animals to generate lin-15; hpEx1421 animals.

\section{Plasmids}

The L123A mutation was engineered by oligonucleotide site-directed mutagenesis in a sad-1 genomic DNA fragment to generate plasmid pBNL32. pJH685 $\left(P_{\text {sad }-1}\right.$-sad$1(L 123 A)$ ) was generated by sub-cloning the NheI/XmaI fragment of pBNL32 into pJH630, which contains the minimal rescuing region (SacII/AvrII fragment) from the cosmid F15A2. pJH73 was generated by inserting a sad-1 mini-gene fragment from pJH56 under the unc-115 promoter into the pBSK vector using the NotI and BamHI sites. pJH1360 was generated by inserting a sad-1 minigene fragment from pJH56 into the HS pPD118.26 vector using the BamHI and ApaI sites. pJH1368 was generated by cloning $g f p$ from pJH21 into the 5 ' end of $s a d-1$ in pJH1360 using the BamHI site.

\section{Protein purification and kinase assays}

Wild-type and L123A versions of SAD-1 cDNA were subcloned into pGEX6p-1. Proteins were expressed in E. coli BL21 by inducing with $0.1 \mathrm{mM}$ isopropyl beta-D-thiogalactoside (IPTG) for 24 hours at room temperature with aeration. Purification was performed as described in [25] except that elution was performed using PreScission protease (GE Healthcare, Buckinghamshire, England) according to the manufacturer's specifications. Eluted protein was snap-frozen at $-80^{\circ} \mathrm{C}$ until use. Due to low yields of protein, quantification and normalization of the wildtype and L123A proteins was performed by western blot using an anti-SAD-1 antibody previously described [21].

For kinase assays, an activation reaction was first performed to phosphorylate SAD-1 at the activation loop threonine, a modification required for full activity of AMP-activated protein kinase (AMPK) family members [25]. In this reaction, approximately $5 \mu \mathrm{g}$ of wild-type or L123A SAD-1 was incubated with $100 \mathrm{ng}$ of LKB1 complex (Upstate, Billerica, MA, USA) at room temperature for 30 minutes in a kinase buffer (KB) containing $25 \mathrm{mM}$ Tris-HCl, $10 \mathrm{mM} \mathrm{MgOAc,} 1 \mathrm{mM}$ dithiothreitol, 0.1\% (v/ v) Triton $\mathrm{X}-100$, and $0.1 \mathrm{mg} / \mathrm{ml}$ bovine serum albumin, $\mathrm{pH} 7.4$ plus $100 \mu \mathrm{M}$ ATP in a volume of $50 \mu \mathrm{L}$. SAD-1 kinase assays were performed using purified tau as a substrate: $400 \mathrm{ng}$ of purified 4R tau (a kind gift of MA Glicks- man, Brigham \& Women's Hospital, Cambridge, MA, USA) diluted in TBS was added to each well of a 96-well tissue culture dish (standard tissue culture treated polystyrene; Corning, Lowell, MA, USA) followed by overnight incubation at $4{ }^{\circ} \mathrm{C}$. The wells were washed with four changes of TBS with $0.1 \%$ Tween-20 (TBS-T) and were then equilibrated with $\mathrm{KB}$ prior to the start of the assay. Immediately prior to the start of the assay, the $\mathrm{KB}$ was aspirated and $10 \mu \mathrm{L}$ of $\mathrm{KB}$ containing the desired concentrations of DMSO/1NA-PP1 (final DMSO concentration was $3.3 \%$ ) was added. The activation reaction was diluted in the same buffer, but the ATP concentration was adjusted to $500 \mu \mathrm{M}$, and $20 \mu \mathrm{L}$ aliquots were added to the wells (30 $\mu \mathrm{L}$ final reaction volume). Reactions, performed in triplicate, were incubated at room temperature and were stopped after 30 minutes by aspirating the liquid and adding $50 \mu \mathrm{L}$ of $10 \mathrm{mM}$ EDTA. To measure phosphorylation of immobilized tau at Ser262, an ELISA was performed using an anti-phosphoTau [S262] antibody (1:1000; Stressgen, Ann Arbor, MI, USA) and a horseradish peroxidase-conjugated goat anti-rabbit antibody (1:25,000; Jackson Immunoresearch, West Grove, PA, USA). Antibodies were diluted in Superblock TBS blocking solution (Pierce, Rockford, IL, USA), and bound antibody was visualized using TMB liquid chromogenic substrate (Sigma-Aldrich, St Louis, MO, USA). After sufficient signal developed, the reactions were stopped by addition of an equal volume of $1 \mathrm{~N} \mathrm{HCl}$, and the plates were read in a Spectramax 384 plate reader. Background signal was determined by measurements from multiple wells that did not contain kinase reactions.

\section{Observation of juls I puncta in VD neurons}

As there is no VD-specific promoter, selective visualization of juIs1 puncta in VD neurons was achieved by inhibiting expression of the juIs1 marker in DD neurons as previously described $[21,27]$. Briefly, double-stranded RNA against the unc-30 transcription factor - which activates the unc-25 promoter used to express the juIs 1 marker - was synthesized from pJH573 as previously described [36] and injected at $100 \mathrm{ng} / \mu \mathrm{L}$ into adult animals carrying the juIs1 marker [27]. Progenies of the injected animals that retained the GFP signal in all 13 of the VD neuron cell bodies but in none of the six DD neuron cell bodies were scored.

\section{DMSO and INA-PPI treatments in C. elegans}

Animals were exposed to DMSO (Sigma-Aldrich, Oakville, Ontario, Canada) or 1NA-PP1 in liquid culture. Overnight LB cultures of OP50 E. coli were divided into $500 \mu \mathrm{L}$ aliquots, and pellets were collected and stored at $20^{\circ} \mathrm{C}$ until use. Each pellet was resuspended in $650 \mu \mathrm{L}$ of S-medium. Animals were incubated in $150 \mu \mathrm{L}$ of the OP50 E. coli resuspension containing $0.33 \%$ DMSO or 33 $\mu \mathrm{M} 1 \mathrm{NA}-\mathrm{PP} 1 / 0.33 \%$ DMSO added to the wells of a $96-$ 
well tissue culture dish (Falcon, St Louis, MO, USA). Exposures throughout the C. elegans life-cycle for validation studies were achieved by treating fourth larval (L4) stage animals and observing their offspring in an adult stage. The solutions were renewed every three days. Exposures until earlier than the adult stage were performed by treating $\mathrm{L} 4$ animals and transferring their offspring onto OP50 E. coli-seeded agar plates at desired stages, assessed by the stage of gonad development. Early first larval (L1) stage-arrested animals were obtained by lysing gravid adult animals in hypochlorite on unseeded agar plates and allowing them to develop at $22^{\circ} \mathrm{C}$ for $16-18$ hours. Late second larval stage (L2) animals were recovered by transferring and keeping early L1-arrested animals on OP50 E. coli-seeded agar plates for 30 hours at $22^{\circ} \mathrm{C}$. Detailed procedures on the incubations are described in Additional files 1 and 2 .

\section{Heat-shock treatments}

All HS treatments were performed by incubating animals at $30^{\circ} \mathrm{C}$ for 7 hours. A gradual decrease in the GFP::SAD1 protein expression level, reflected by the intensity of GFP fluorescence, was observed following HS over the course of a day. Therefore, for post-L2 HS treatments, late L2 animals obtained as described above were heatshocked twice, 15 hours apart.

\section{Puncta quantification and statistical analyses}

Significance-testing employed paired $t$-test for the in vitro kinase assay and the Wilcoxon rank-sum test for the VD neuron analyses as implemented in the R statistical environment (v2.6.2) [37]. juIs1 puncta were quantified by an in-house developed program called 'punctaanalyser' using the MatLab software (v6.5; Mathworks, Inc., Natick, MA, USA) and analyzed using kernel density estimation implemented in $\mathrm{R}$ (v2.6.2).

\section{Abbreviations}

C. elegans: Caenorhabditis elegans; GFP: green fluorescent protein; HS: heat shock; LOF: loss-of-function; SAD: Synapses of amphids defective; ts: temperature sensitive; SAD-1as: analog-sensitive version of SAD-1 kinase.

\section{Competing interests}

The authors declare that they have no competing interests.

\section{Authors' contributions}

JSMK designed and conducted C. elegans experiments, analyzed data, prepared figures, and wrote the manuscript. BNL conceived the study, designed and performed in vitro experiments, analyzed data, and prepared figures. CZ and KMS supplied 1NA-PP1. BNL, JRS, and MZ edited the manuscript. JRS and $M Z$ supervised the work. All authors read and approved the manuscript.

\section{Additional material}

\section{Additional file 1}

Experimental system and different exposures (1). Exposures to DMSO and/or 1NA-PP1. Each flow corresponds to a time-course as indicated in square boxes. L4 animals were exposed to DMSO/1NA-PP1, and their progenies were transferred to agar plates at different stages to be observed immediately or at an adult stage. Full lifetime exposures required four days of incubation; solutions were renewed after three days.

Click here for file

[http://www.biomedcentral.com/content/supplementary/1749-

8104-3-23-S1.pdf]

\section{Additional file 2}

Experimental system and different exposures (2). Exposures to DMSO and/or 1NA-PP1. Each flow corresponds to a time-course as indicated in square boxes. Gravid adult animals were sacrificed to obtain synchronized populations. Synchronized animals were exposed to DMSO/1NA-PP1 during different larval stages and observed immediately or at an adult stage.

Click here for file

[http://www.biomedcentral.com/content/supplementary/17498104-3-23-S2.pdf]

\section{Acknowledgements}

We thank MA Glicksman for tau protein; $K$ Shen for the $P_{\text {odr- }}$-gfp injection marker; P Candido and AZ Fire for the PPDI 18.26 vector; C Mok and T Kawano for the 'punctaanalyser' program; PC Boutros for statistical assistance and critical comments on the manuscript; and members of MZ's laboratory for critical review of the manuscript. This work was funded by an NIH grant (ROIAI440099) to KMS, a grant from NIH (NINDS) to JRS, and an NSERC discovery grant ( I | 20339/2) awarded to MZ. JSMK is a recipient of NSERC graduate student fellowships (CGS M and PGS M). BNL is a fellow of the Damon Runyon Cancer Research Foundation (DRG 19|4-06).

\section{References}

I. Ciani L, Krylova O, Smalley MJ, Dale TC, Salinas PC: A divergent canonical WNT-signaling pathway regulates microtubule dynamics: dishevelled signals locally to stabilize microtubules. J Cell Biol 2004, 164:243-253.

2. Klassen MP, Shen K: Wnt signaling positions neuromuscular connectivity by inhibiting synapse formation in $\mathrm{C}$. elegans. Cell 2007, I30:704-716.

3. Ishii N, Wadsworth WG, Stern BD, Culotti JG, Hedgecock EM: UNC-6, a laminin-related protein, guides cell and pioneer axon migrations in C. elegans. Neuron 1992, 9:873-88I.

4. Serafini T, Kennedy TE, Galko MJ, Mirzayan C, Jessell TM, TessierLavigne M: The netrins define a family of axon outgrowth-promoting proteins homologous to C. elegans UNC-6. Cell I994, 78:409-424.

5. Kolodkin AL, Matthes DJ, Goodman CS: The semaphorin genes encode a family of transmembrane and secreted growth cone guidance molecules. Cell 1993, 75:1389-1399.

6. Colon-Ramos DA, Margeta MA, Shen K: Glia promote local synaptogenesis through UNC-6 (netrin) signaling in C. elegans. Science 2007, 3 I 8: 103-106.

7. Godenschwege TA, Hu H, Shan-Crofts X, Goodman CS, Murphey RK: Bi-directional signaling by Semaphorin I a during central synapse formation in Drosophila. Nat Neurosci 2002, 5: I294-|30|.

8. Wang X, Weiner JA, Levi S, Craig AM, Bradley A, Sanes JR: Gamma protocadherins are required for survival of spinal interneurons. Neuron 2002, 36:843-854. 
9. Weiner JA, Wang X, Tapia JC, Sanes JR: Gamma protocadherins are required for synaptic development in the spinal cord. Proc Natl Acad Sci USA 2005, 102:8-14.

10. Knight ZA, Shokat KM: Chemical genetics: where genetics and pharmacology meet. Cell 2007, I 28:425-430.

II. Hanke JH, Gardner JP, Dow RL, Changelian PS, Brissette WH, Weringer EJ, Pollok BA, Connelly PA: Discovery of a novel, potent, and Src family-selective tyrosine kinase inhibitor. Study of Lck- and FynT-dependent T cell activation. J Biol Chem 1996, 271:695-701.

12. Anthony C, Bishop C-yK, Shah K, Witucki L, Kevan M, Shokat, Liu Yi: Generation of monospecific nanomolar tyrosine kinase inhibitors via a chemical genetic approach. J Am Chem Soc |999, I 2 |:627-63|.

13. Bishop AC, Ubersax JA, Petsch DT, Matheos DP, Gray NS, Blethrow J, Shimizu E, Tsien JZ, Schultz PG, Rose MD, et al.: A chemical switch for inhibitor-sensitive alleles of any protein kinase. Nature 2000, 407:395-40I.

14. Wang H, Shimizu E, Tang YP, Cho M, Kyin M, Zuo W, Robinson DA, Alaimo PJ, Zhang C, Morimoto H, et al.: Inducible protein knockout reveals temporal requirement of CaMKII reactivation for memory consolidation in the brain. Proc Natl Acad Sci USA 2003, 100:4287-4292.

15. Wan L, de los Santos T, Zhang C, Shokat K, Hollingsworth NM: Mek I kinase activity functions downstream of REDI in the regulation of meiotic double strand break repair in budding yeast. Mol Biol Cell 2004, 15: I I-23.

16. Chen $\mathrm{X}, \mathrm{Ye} \mathrm{H}$, Kuruvilla R, Ramanan N, Scangos KW, Zhang C, Johnson NM, England PM, Shokat KM, Ginty DD: A chemical-genetic approach to studying neurotrophin signaling. Neuron 2005 , 46:|3-2|.

17. Brodersen P, Petersen M, Bjorn Nielsen H, Zhu S, Newman MA, Shokat KM, Rietz S, Parker J, Mundy J: Arabidopsis MAP kinase 4 regulates salicylic acid- and jasmonic acid/ethylene-dependent responses via EDSI and PAD4. Plant J 2006, 47:532-546.

18. Gregan J, Zhang C, Rumpf C, Cipak L, Li Z, Uluocak P, Nasmyth K, Shokat KM: Construction of conditional analog-sensitive kinase alleles in the fission yeast Schizosaccharomyces pombe. Nat Protoc 2007, 2:2996-3000.

19. Burkard ME, Randall CL, Larochelle S, Zhang C, Shokat KM, Fisher RP, Jallepalli PV: Chemical genetics reveals the requirement for Polo-like kinase I activity in positioning RhoA and triggering cytokinesis in human cells. Proc Natl Acad Sci USA 2007, 104:4383-4388.

20. Crump JG, Zhen M, Jin Y, Bargmann Cl: The SAD-I kinase regulates presynaptic vesicle clustering and axon termination. Neuron 2001, 29: II5-129.

21. Hung W, Hwang C, Po MD, Zhen M: Neuronal polarity is regulated by a direct interaction between a scaffolding protein, Neurabin, and a presynaptic SAD-I kinase in Caenorhabditis elegans. Development 2007, 134:237-249.

22. Kishi M, Pan YA, Crump JG, Sanes JR: Mammalian SAD kinases are required for neuronal polarization. Science 2005 307:929-932.

23. Barnes AP, Lilley BN, Pan YA, Plummer LJ, Powell AW, Raines AN, Sanes JR, Polleux F: LKB I and SAD kinases define a pathway required for the polarization of cortical neurons. Cell 2007, 1 29:549-563.

24. Inoue E, Mochida S, Takagi H, Higa S, Deguchi-Tawarada M, TakaoRikitsu E, Inoue M, Yao I, Takeuchi K, Kitajima I, et al:: SAD: a presynaptic kinase associated with synaptic vesicles and the active zone cytomatrix that regulates neurotransmitter release. Neuron 2006, 50:26I-275.

25. Lizcano JM, Goransson O, Toth R, Deak M, Morrice NA, Boudeau J, Hawley SA, Udd L, Makela TP, Hardie DG, et al: LKB I is a master kinase that activates 13 kinases of the AMPK subfamily, including MARK/PAR-I. EMBO / 2004, 23:833-843.

26. Zhen $M$, Jin Y: The liprin protein SYD-2 regulates the differentiation of presynaptic termini in C. elegans. Nature 1999 40I:37I-375.

27. Hallam SJ, Goncharov A, McEwen J, Baran R, Jin Y: SYD-I, a presynaptic protein with PDZ, C2 and rhoGAP-like domains, specifies axon identity in C. elegans. Nat Neurosci 2002, 5:1137-11146.
28. White JG, Albertson DG, Anness MA: Connectivity changes in a class of motoneurone during the development of a nematode. Nature 1978, 271:764-766.

29. Yeh E, Kawano T, Weimer RM, Bessereau JL, Zhen M: Identification of genes involved in synaptogenesis using a fluorescent active zone marker in Caenorhabditis elegans. J Neurosci 2005, 25:3833-384I.

30. Fire A, Harrison SW, Dixon D: A modular set of lacZ fusion vectors for studying gene expression in Caenorhabditis elegans. Gene 1990, 93:189-198.

31. Holt JR, Gillespie SK, Provance DW, Shah K, Shokat KM, Corey DP, Mercer JA, Gillespie PG: A chemical-genetic strategy implicates myosin- I $\mathrm{c}$ in adaptation by hair cells. Cell 2002, 108:37I-38I.

32. Witte $H$, Neukirchen D, Bradke F: Microtubule stabilization specifies initial neuronal polarization. J Cell Biol 2008, 180:619-632.

33. Larochelle S, Batliner J, Gamble MJ, Barboza NM, Kraybill BC, Blethrow JD, Shokat KM, Fisher RP: Dichotomous but stringent substrate selection by the dual-function Cdk7 complex revealed by chemical genetics. Nat Struct Mol Biol 2006, 13:55-62.

34. Blethrow JD, Glavy JS, Morgan DO, Shokat KM: Covalent capture of kinase-specific phosphopeptides reveals CdkI-cyclin B substrates. Proc Natl Acad Sci USA 2008, 105: | 442-| 447.

35. Brenner S: The genetics of Caenorhabditis elegans. Genetics 1974, 77:7|-94.

36. Fire A, Xu S, Montgomery MK, Kostas SA, Driver SE, Mello CC: Potent and specific genetic interference by double-stranded RNA in Caenorhabditis elegans. Nature 1998, 391:806-8I I.

37. The R Foundation for Statistical Computing [http://www.rproject.org/]

Publish with Bio Med Central and every scientist can read your work free of charge

"BioMed Central will be the most significant development for disseminating the results of biomedical research in our lifetime. "

Sir Paul Nurse, Cancer Research UK

Your research papers will be:

- available free of charge to the entire biomedical community

- peer reviewed and published immediately upon acceptance

- cited in PubMed and archived on PubMed Central

- yours - you keep the copyright
BioMedcentral 